\title{
Descripción Histológica de las Glándulas Anexas del Aparato Digestivo de Avestruz (Struthio camelus var. domesticus)
}

\author{
Histologic Description of the Annexed Glands from the \\ Ostrich Digestive System (Struthio camelus var. domesticus) \\ *,**Illanes, J.; **Fertilio, B.; ***uijada, M.; "Leyton, V. \& "Verdugo, F.
}

ILLANES, J.; FERTILIO, B.; QUIJADA, M.; LEYTON, V. \& VERDUGO, F. Descripción histológica de las glándulas anexas del aparato digestivo de Struthio camelus var. domesticus. Int. J. Morphol., 24(3):297-302, 2006.

\begin{abstract}
RESUMEN: En la última década, la crianza del avestruz en Chile ha ido aumentando sustancialmente, por lo cual se ha hecho necesario incrementar los estudios para mejorar la producción de esta especie. La literatura señala sólo estudios sobre la fisiología y anatomía de esta especie. Nuestro objetivo es aportar al conocimiento de la histología normal de las glándulas anexas al aparato digestivo. Esta investigación se realizó con 6 avestruces clínicamente sanas, de las que se obtuvieron muestras representativas del hígado, pro ventrículo y páncreas. Se realizaron cortes histológicos, los que fueron teñidos y montados para su análisis comparativo bajo microscopio de luz, entre avestruz y gallina. La histología de las glándulas anexas del aparato digestivo es semejante a la descrita en la gallina. Sin embargo, en el hígado los cordones de hepatocitos se disponen en forma radial, tanto alrededor de la vena central como de los espacios porta, característica no observada en otras especies. Con respecto al pro ventrículo, en la mucosa se observan glándulas tubulares simples o ramificadas, semejantes a las glándulas fúndicas de los mamíferos. En la submucosa se observan glándulas túbuloalveolares compuestas con células parietales. El páncreas no presenta diferencias destacables.
\end{abstract}

PALABRAS CLAVE: Avestruz; Glándulas; Aparato Digestivo.

\section{INTRODUCTION}

El avestruz pertenece al grupo de los Ratite del que se conocen varios Órdenes: entre ellos el Orden Struthioniformes, al cual pertenece el avestruz que habita en desiertos y sabanas de África y la península Arábiga. En América del Sur existe el Orden Rheiforme (ñandúes). En Australia está el Orden Casuariformes, (casuarios y emues). Otro Orden lo conforman los Tinamiformes (tinamúes y perdices del monte), constituyendo el grupo más numeroso y, a la vez, el menos semejante a los otros, que habitan en zonas tropicales desde México hasta Argentina (Navarro, \& Benitez, 2003). El avestruz o "ave camello", debido a su similitud con los dromedarios, fue denominada por Linnaeus en 1758, como Struthio camelus. Se describen cuatro subespecies, S. c. camelus, S. c. molybdophanes, S. c. massaicus y S. c. australis, todas originarias de África y ocupando extensas áreas geográficas (Deeming, 2001).

Swart et al. (1987) describió la variedad domesticus, que fue desarrollada mediante programas de mejora genética, caracterizándose por su pequeña talla, una estructura del plu- maje bien desarrollada, un carácter dócil, facilitando su crianza en granja, a la que se conoce como avestruz de cuello negro o African Black (Deeming). Todos los estudios que se han realizado, están enfocados a esta subespecie y es la que se ocupa actualmente para la producción de carne y subproductos (Cunnimgham, 1990). El avestruz, es en la actualidad, el ave no voladora de mayor tamaño, alcanzando 2,75 m de alzada y $150 \mathrm{~kg}$ de peso. En el caso de la African Black, el macho adulto presenta un plumaje principalmente negro, con plumas blancas en las alas y en la cola, y presenta el cuello de coloración gris. La hembra presenta un color pardo grisáceo poco llamativo y de color gris y blanco en la cola (Angel, 1996; Deeming). El aparato digestivo presenta glándulas como proventrículo (estómago glandular), hígado y páncreas que han sido descritos anatómicamente. El estómago verdadero del avestruz corresponde al pro ventrículo o estómago glandular, tiene forma de saco y anatómicamente ocupa la parte craneal del abdomen en el hipocondrio izquierdo (Bezuidenhout, 20001), en el que se inicia la digestión de las proteínas mediante la secreción de

\footnotetext{
* Facultad de Medicina. Clínica Alemana-Universidad del Desarrollo, Chile.

${ }^{* *}$ Facultad de Medicina Veterinaria. Universidad Iberoamericana de Ciencia y Tecnología, Chile.
} 
pepsinógeno y ácido clorhídrico (Camiruaga \& Simoneti, 2003). Por otro lado, debido al creciente interés en la explotación de sus productos, se ha hecho necesario investigar para solucionar los problemas de producción del avestruz dentro de una ganadería moderna. La histología en esta especie no ha sido muy estudiada, lo que ha impedido, en cierto modo, conocer y satisfacer con mayor certeza sus requerimientos nutricionales (Camiruaga \& Simoneti).

\section{MATERIAL Y MÉTODO}

El material biológico empleado en este trabajo, corresponde a muestras de glándulas asociadas al aparato digestivo, obtenidas desde 6 avestruces adultas, clínicamente sanas, con un rango de 12 a 14 meses de edad, provenientes de criaderos de la Sexta Región de Chile. El material se obtuvo del frigorífico FAENAGRO ubicado en Graneros, ruta 5 sur, km. 74, VI Región, Chile. Obtenidas las muestras de las diferentes glándulas asociadas al aparato digestivo del avestruz: proventrículo, hígado y páncreas, fueron fijadas en formalina tamponada al $10 \%$ y luego se siguió con el procedimiento habitual de técnica histológica. Cortes de $5 \mu \mathrm{m}$ de grosor, fueron observados, fotografiados y analizados bajo microscopio de luz (Olympus BX41 con cámara digital Olympus C-5060 Wide zoom, 5.1 mega pixel). Con las fotografías, se realizó un análisis comparativo de los aspectos histológicos encontrados en avestruz, con aquellos descritos en otras especies.

\section{RESULTADOS}

Hígado: El hígado es una glándula de carácter exocrino y endocrino, de color rojo pardo que presenta 4 lóbulos, situado anatómicamente en el hipocondrio derecho (Figura 1). El parénquima está tabicado de modo irregular y constituidos por cordones de hepatocitos de disposición radiada respecto a una vena central. Los hepatocitos generalmente presentan uno o dos núcleos. Entre los cordones se encontraron capilares sinusoides, donde se observan macrófagos. Cabe señalar que los hepatocitos ubicados alrededor de los espacios porta, también se disponían en forma radial (Figs. 1a, $1 b, 1 \mathrm{c}$ y 1d). El estroma de tejido conjuntivo denso forma la cápsula, espacios porta (tabiques) y el hilio del órgano. (Figs. 1e, 1f y $1 \mathrm{~g}$ ). Desde la cápsula nacen los tabiques que dividen el órgano de modo irregular (Figs. 1f y $1 \mathrm{~g}$ ). El espacio porta corresponde al tejido conectivo laxo que contiene vasos sanguíneos derivados de la vena porta y de la arteria hepática, vasos linfáticos, conductos biliares, éstos últimos presentan epitelio de revestimiento cúbico simple (Figs. if y $1 \mathrm{~g})$.
Proventrículo (Mucosa y Submucosa): El proventrículo del avestruz es un órgano que presenta un lumen amplio y en su pared se reconocen 4 capas: mucosa, submucosa, muscular y serosa. La capa mucosa presenta glándulas tubulares simples y ramificadas. La capa submucosa presenta glándulas túbuloalveolares compuestas (Figs. 2 y 2a). La mucosa presenta gran cantidad de vellosidades, entre cuyas bases se ubican las criptas donde vierten su secreción las glándulas tubulares simples o ramificadas secretoras de pepsinógeno y ácido clorhídrico (Figs. 2a, 2b, 2c, y 2d). El epitelio de revestimiento es prismático simple en su superficie, la que está cubierta de mucus (glicocáliz). En las criptas es de tipo cúbico simple, con gran capacidad regenerativa. Bajo el epitelio de las vellosidades y entre las glándulas, se encuentra el corion de tejido conectivo laxo con gran infiltración de linfocitos. Bajo la mucosa se encuentra la muscular de la mucosa de tejido muscular liso, no bien definida y que emite tabiques hacia la submucosa (Fig. 2c). La submucosa es gruesa y está conformada por tejido conectivo denso que emite tabiques alrededor de las glándulas tubuloalveolares compuestas, constituyendo lobulillos formados por conglomerados de adenómeros túbuloalveolares alrededor de un lumen central (Figs. 2a, 2b, 2c, 2e y 2f). Las glándulas vierten su secreción al lumen del pro ventrículo, mediante su conducto excretor revestido por epitelio cilíndrico simple. Entre las células secretoras de forma cúbica y cilíndrica de los adenómeros, son abundantes las parietales, productoras de pepsinógeno y ácido clorhídrico. En la submucosa del proventrículo se observan también células ganglionares de los plexos submucosos.

Páncreas: El páncreas del avestruz es una glándula extensa, muy lobulillada, de color rosado pálido y rodeada por tejido adiposo (Fig. 3). Se clasifica como una glándula mixta y compuesta, constituida por un parénquima (islotes pancreáticos y acinos glandulares) y un estroma (cápsula, tabiques e hilio).

El parénquima exocrino está representado por adenómeros túbuloacinares compuestos de secreción serosa y agrupaciones celulares muy irrigadas, que corresponden a los islotes pancreáticos que cumplen la función endocrina (Figs. 3a, 3b, 3c y 3d). Las células que constituyen los adenómeros son prismáticas con un núcleo redondo situado en el tercio basal. La afinidad tintorial de esta zona es basófila y la apical es acidófila. En el lumen de los acinos se observan células centro-acinosas que corresponden al inicio de los conductos excretores (Figs. 3b, 3d y 3e).

El estroma está conformado por la cápsula que rodea al órgano, los tabiques y el hilio. La cápsula, es relativamente delgada constituida por tejido conectivo laxo que forma tabiques hacia el interior del parénquima, dividiéndolo en pequeños lóbulos y lobulillos. 


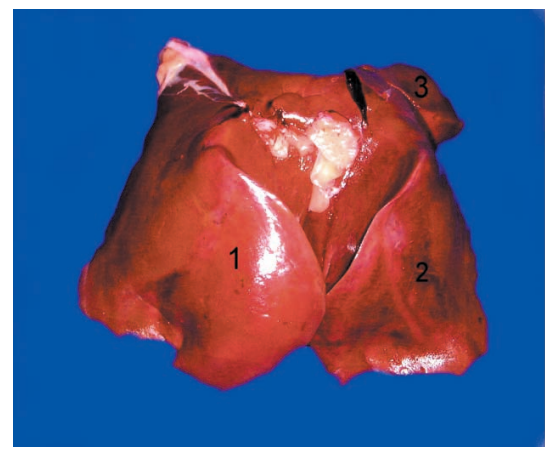

Fig. 1. Aspecto anatómico del hígado de avestruz. Muestra 3 de sus 4 lóbulos (1, 2 y $3)$.

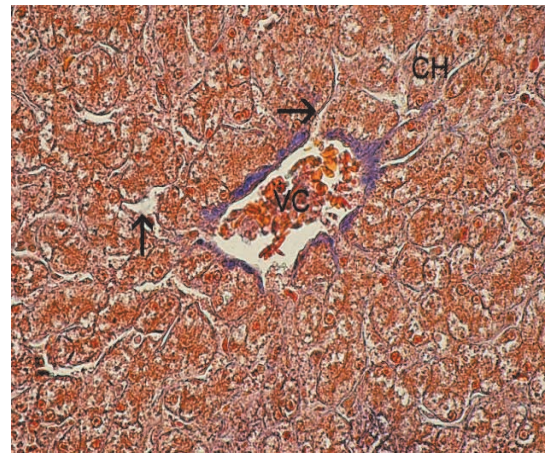

Fig. 1c. Corte histológico de la vena central de un lobulillo hepático. VC. Vena central del lobulillo; $\mathrm{CH}$. Cordones de hepatocitos, capilares sinusoides que drenan en la vena central (flecha). Tricrómico de Mallory. 400X.

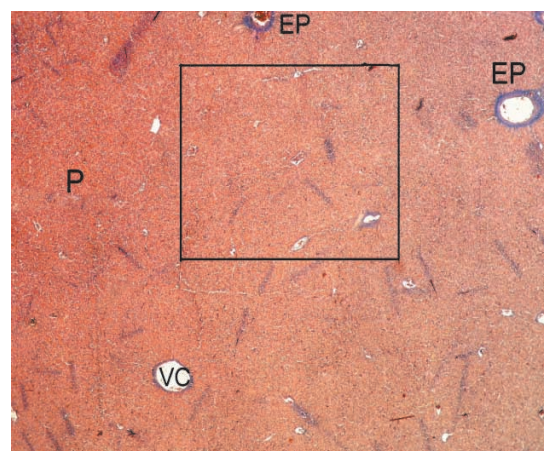

Fig. 1a. Corte histológico panorámico del hígado de avestruz. VC. Vena central; EP. Espacio porta; P. Parénquima hepático. El recuadro se muestra a mayor aumento en la Fig. 1b. Tricrómico de Mallory. 40X.

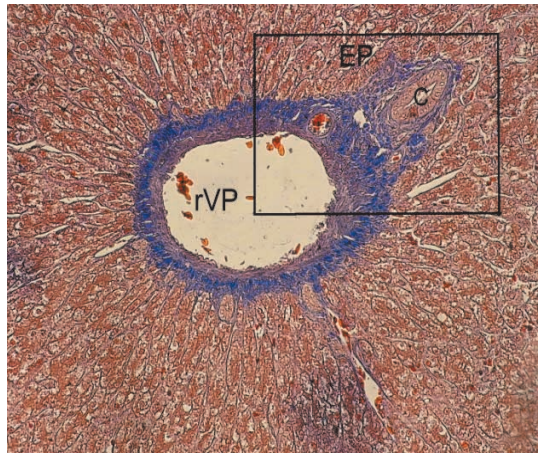

Fig. 1d. Corte histológico de un espacio porta. EP. Espacio porta; rVP. Rama de la vena porta; C. Conducto biliar. Nótese la disposición radiada de los hepatocitos en torno al espacio porta. El recuadro se muestra a mayor aumento en la Fig. 1e. Tricrómico de Mallory. 200X.

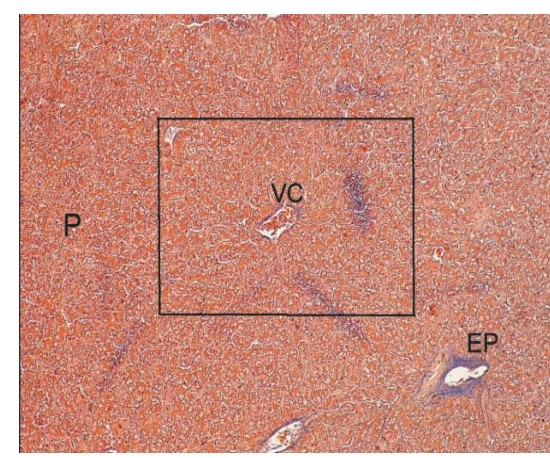

Fig. 1b. Corte histológico de un lobulillo hepático. VC.Vena central del lobulillo; P. Parénquima hepático; EP. Espacio porta. El recuadro se muestra a mayor aumento en la Fig. 1c. Tricrómico de Mallory. 100x.

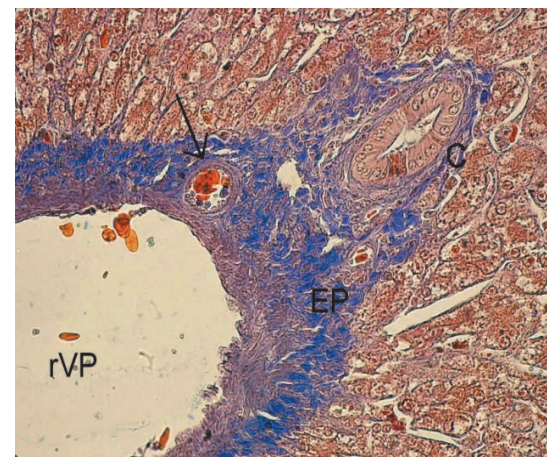

Fig. 1e. Corte histológico de un espacio porta. rVP. Rama de la vena porta; Rama de la arteria hepática (flecha), conducto biliar (C). Tricrómico de Mallory. 400X.

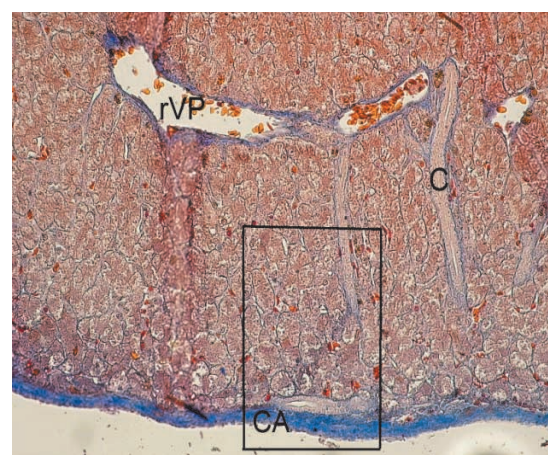

Fig. 1f. Corte histológico que muestra la cápsula del hígado. rVP. Rama de la vena porta; C. Conducto biliar, CA. Cápsula (CA). El recuadro se muestra a mayor aumento en la Fig. 1g. Tricrómico de Mallory. 200X.

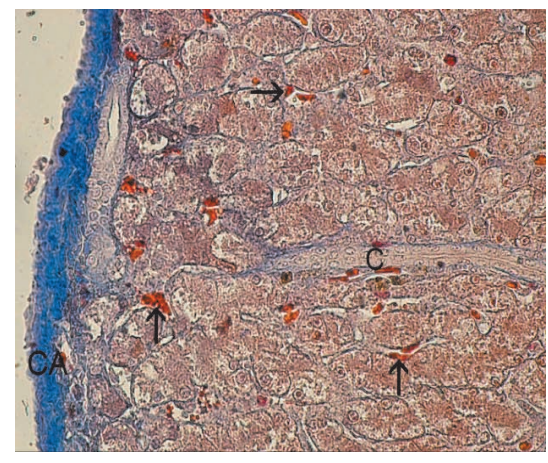

Fig. 1g. Corte histológico que muestra la cápsula del hígado. CA. Cápsula; C. Conducto biliar; capilares sinusoides (flechas). Tricrómico de Mallory. 400X. 


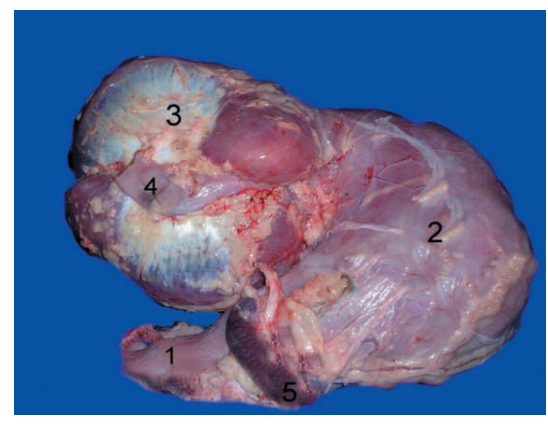

Fig. 2. Aspecto anatómico del proventrículo de avestruz y su relación con otros órganos. 1.Porción final del esófago; 2. Proventrículo; 3 . Estómago muscular (molleja); 4. Inicio del duodeno; 5. Bazo.

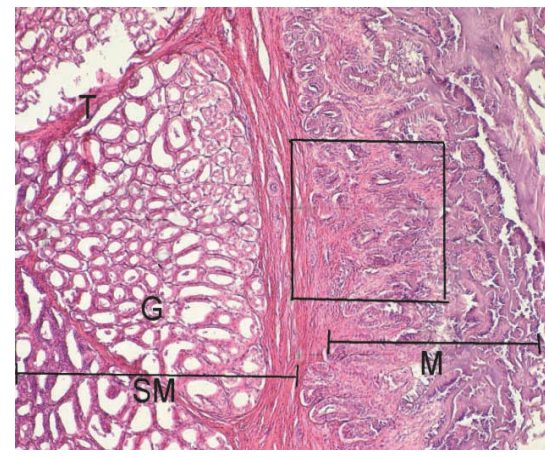

Fig. 2c. Corte histológico de la pared del proventrículo del avestruz. Mucosa (M), glándulas tubulares simples y ramificadas de la mucosa. SM. Submucosa; G. Glándulas túbuloalveolares de la submucosa; T. Tabiques. El recuadro se muestra a mayor aumento en la Fig. 2d. H-E. 100X.

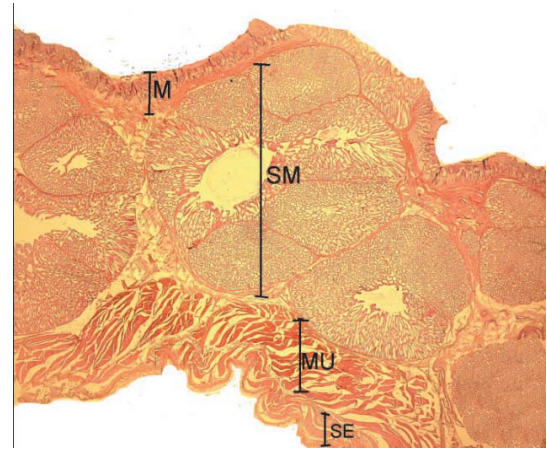

Fig. 2a. Corte panorámico del pro ventrículo de avestruz. M. Mucosa; SM. Submucosa; M. Muscular interna; ME. Muscular externa. H-E. 12.5X.

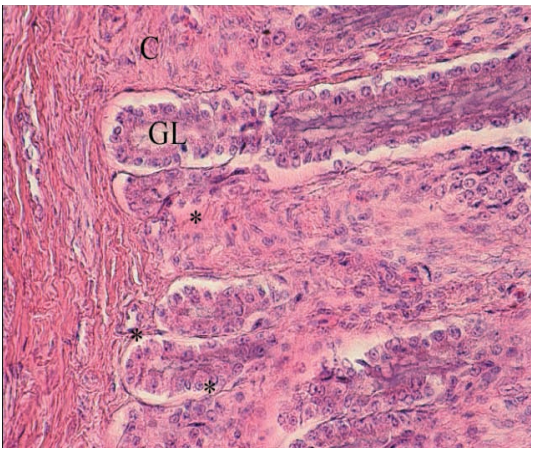

Fig. 2d. Corte histológico de la mucosa del pro ventrículo. GL. Glándulas fúndicas en corte longitudinal; Célula parietal (flechas) y C. Corion. H-E. 200X.

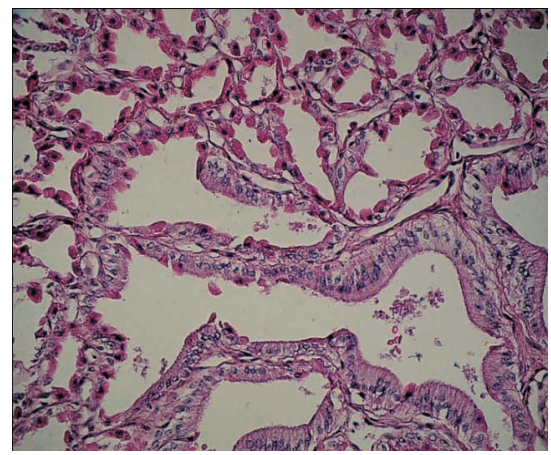

Fig. 2f. Corte histológico de la submucosa del proventrículo. Muestra el lumen de un conducto excretor de las glándulas de la submucosa y adenómeros túbuloalveolares con células parietales. H-E. 400X.

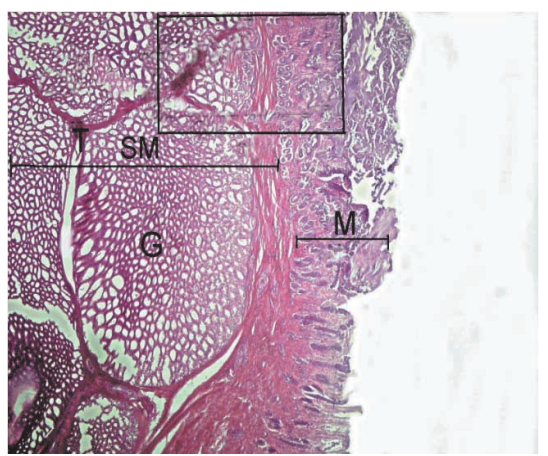

Fig. 2b. Corte histológico de la pared del pro ventrículo. M. Mucosa; SM. Submucosa; G. Glándulas tubuloalveolares compuestas de la submucosa; T. Tabiques. El recuadro se muestra a mayor aumento en la Fig. 2c. H-E. 40X.

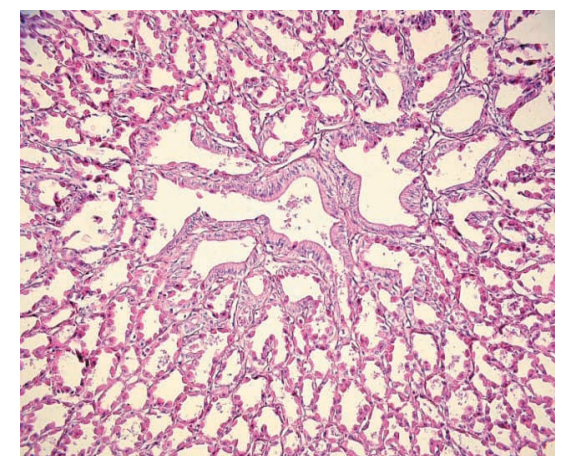

Fig. 2e. Corte histológico de la submucosa del proventrículo. Muestra el lumen de un conducto excretor y adenómeros túbuloalveolares de las glándulas submucosas compuestas que se vacían en él. H-E. 200X. 


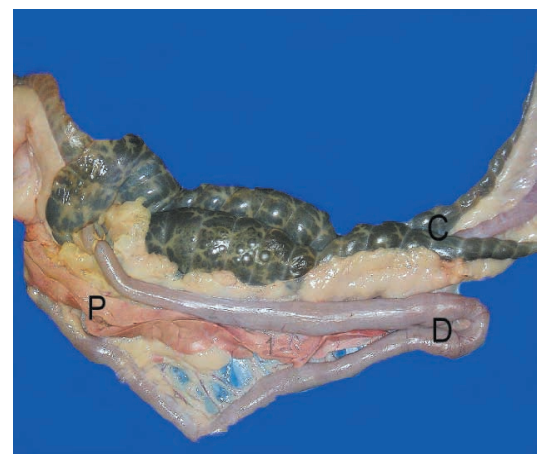

Fig. 3. Aspecto anatómico del páncreas y sus órganos asociados, en el avestruz. P. Páncreas; D. Duodeno; C. Bifurcación del ciego.

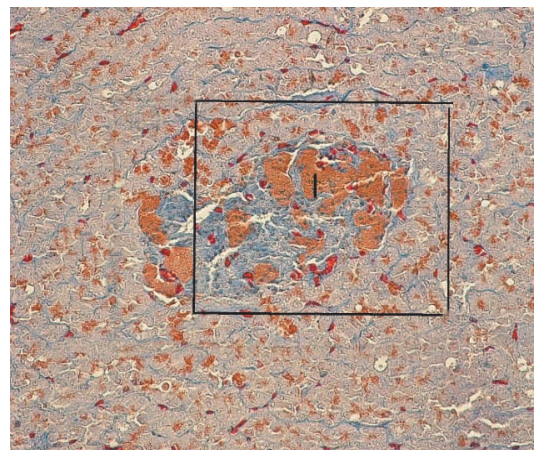

Fig. 3c. Corte histológico de un islote pancreático. I. Islote pancreático. El recuadro se muestra a mayor aumento en la Fig. 3d. Tricrómico de Mallory. 200X.

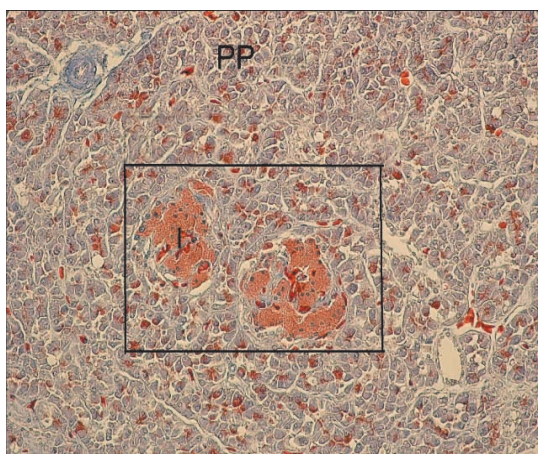

Fig. 3a. Corte histológico del parénquima pancreático, en el avestruz. I. Islotes pancreáticos; PP. Parénquima pancreático. El recuadro se muestra a mayor aumento en la Fig. 3b. Tricrómico de Mallory. 200X

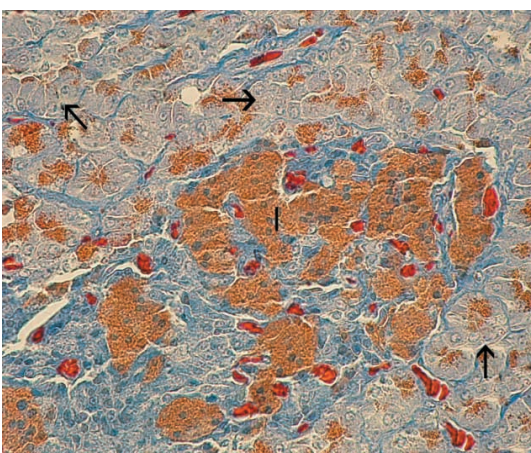

Fig. 3d. Corte histológico de un islote pancreático. I. Islote pancreático, Acino seroso (flechas). Tricrómico de Mallory. $400 X$.

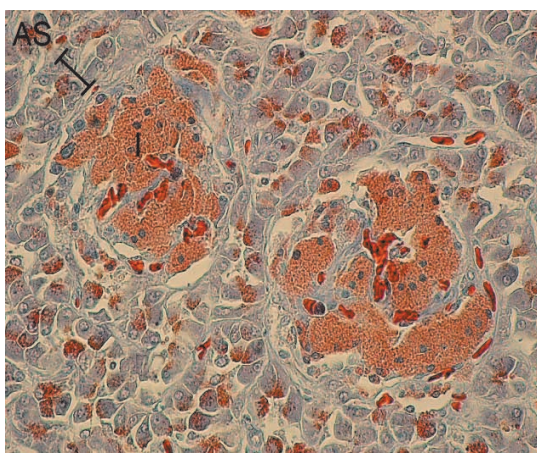

Fig. 3b. Corte histológico de un islote pancreático. I. Islotes pancreáticos; AS. Acino seroso. Nótese la irrigación del islote pancreático. Tricrómico de Mallory. $400 X$.

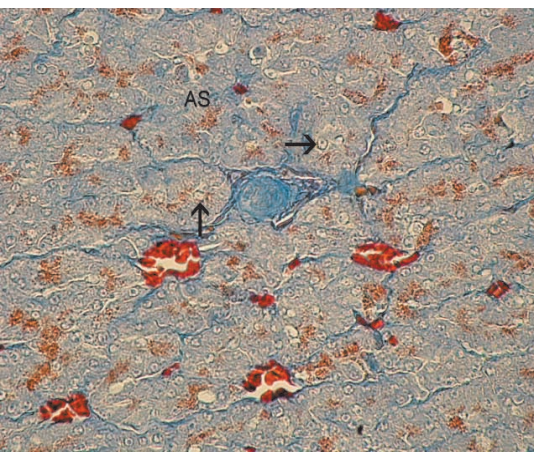

Fig. 3e. Corte histológico del parénquima pancreático. AS. Acino seroso; Célula centroacinosa (flechas). Tricrómico de Mallory 400X.

\section{DISCUSIÓN}

Histológicamente, el hígado del avestruz no presenta diferencias significativas con el hígado de la gallina y de otras especies (Bacha \& Wood, 1991). Sin embargo, la disposición de los cordones de hepatocitos, tanto alrededor de la vena central como de los espacios porta, es radiada. Se observan muy bien los espacios porta y venas centrales; sin embargo, no se observa una clara organización en lobulillos. La disposición de capilares sinusoides en ambas especies, es en la periferia de los cordones de hepatocitos. La cápsula es de tejido conectivo denso, de gran firmeza y resistencia. Los espacios portales muestran conductos biliares que, en ambas especies, presentan un epitelio de revestimiento cúbico simple. Estos espacios portales están conformados de tejido conectivo laxo, en ambas especies, donde encontramos una o más ramas de la arteria hepática y ramas de la vena porta, uno o más conductos biliares, y vasos linfáticos (Bacha \& Wood). Con respecto al proventrículo del avestruz, podemos señalar que no presenta grandes diferencias con el de la gallina. (Bacha \&
Wood; McLelland, 1992). La mucosa del órgano, en ambas especies, presenta una gran cantidad de vellosidades con un epitelio de revestimiento prismático simple. En ella, encontramos los conductos excretores de tipo primario que presentan un epitelio prismático simple de las glándulas túbuloalveolares compuestas de la submucosa, los que en ambas especies desembocan en el lumen del proventrículo (Bacha \& Wood; McLelland). Si bien las glándulas tubulares simples y/o ramificadas de la mucosa son poco desarrolladas, son muy semejantes a las glándulas fúndicas de los mamíferos monogástricos (perro) y rumiantes no verdaderos (camélidos) (Illanes, et al. 1994; Cummings et al., 1972). La submucosa de avestruz y gallina es similar, presenta glándulas túbulo-alveolares compuestas y la secreción de éstas se libera al lumen mediante conductos excretores. Cabe destacar, que estas glándulas presentan gran cantidad de células parietales secretoras de ácido clorhídrico. (Bacha \& Wood; McLelland). Con respecto al páncreas tanto del avestruz como 
de la gallina, presenta adenómeros de tipo túbulo-acinar compuestos de secreción serosa. La cápsula que rodea al órgano, de tejido conectivo laxo, es más notoria en el avestruz que en la gallina (Bacha \& Wood). Las células que constituyen los adenómeros, en ambas especies son del tipo prismático, con un núcleo redondo de gran tamaño, que se ubica en la zona basal. En el lumen se observan células centroacinosas. Como en todas la especies, la función endocrina del órgano está dada por los islotes pancreáticos que se ubican como agrupaciones celulares muy irrigadas entre los acinos serosos, aparentemente más numerosos en la gallina que en el avestruz. En ambas especies, los islotes pancreáticos están rodeados de tejido conectivo laxo con abundante irrigación. (Bacha \& Wood). Sin embargo, en el avestruz los islotes pancreáticos son de mayor tamaño. El estroma de tejido conectivo laxo del órgano, como en la mayoría de las especies, está constituido por la cápsula, tabiques y el hilio. La cápsula del páncreas del avestruz es delgada, constituida de tejido conectivo laxo, en ambas especies. Los tabiques son más notorios en el avestruz que en la gallina, ya que se logra distinguir con mayor claridad el adenómero, lo que no ocurre en la gallina. Este órgano en el avestruz es muy lobulado (Bacha \& Wood). Otras glándulas anexas al tubo digestivo del avestruz son las de carácter mucoso, descritas en toda la extensión de la mucosa del esófago (Illanes et al., 2006)Este análisis histológico es un aporte no sólo a la biología, sino también puede ser de gran utilidad, especialmente para comprender mejor los procesos de carácter alimentario y nutritivo en la crianza de estas aves.

AGRADECIMIENTOS: A Frigorífico FAENAGRO, por su cooperación tanto con el material biológico como su disposición, sin ello no hubiera sido posible este estudio.

ILLANES, J.; FERTILIO, B.; QUIJADA, M.; LEYTON, V. \& VERDUGO, F. Histologic description of the annexed glands from the ostrich digestive system Struthio camelus var. domesticus. Int. J. Morphol., 24(3):297-302, 2006.

SUMMARY: In the last decade the upbringing of the ostrich in Chile has increased substantially, for this reason it is necessary to increase the studies to improve the production of this species. The literature only points out studies on the physiology and anatomy of this species. The purpose of this work is to contribute to the knowledge of the normal histology from the annexed glands to the digestive system. This study was carried out clinically using 6 healthy ostriches, of which representative samples of the liver, proventricle and pancreas. Histological sections were realized, mounted and stained for their comparative analysis under low light microscope to describe with those cited in the hen. The histology of the annexed glands from the digestive system is similar to the described in the hen. However, in the liver the arrangement hepatic cords so is in the central vein such as the portal spaces is radial, characteristic not observed in other species. In relation to the histology of the proventricle, the tubular glands are quite, similar to the fundic glands of the mammals. In the submucosa compound tubulosacular glands are observed, with parietal cells. The pancreas doesn't present prominent differences.

KEY WORDS: Ostrich; Glands; Digestive system.

\section{REFERENCIAS BIBLIOGRÁFICAS}

Angel, C. R. A Review of Ratites Nutrition. Animal Feed Science and Technology, 60:241-6, 1966.

Bacha, W. J. \& Wood, L. M. Aparato Digestivo. En: Atlas color de Histología Veterinaria. Inter-médica, Buenos Aires, 1991. pp. $111-50$.

Bezuidenhout, A. J. Anatomía. En: El Avestruz: Biología, producción y sanidad. Acribia, Zaragoza, 2001. pp 13-50.

Camiruaga, M. \& Simoneti, C. Avestruces, Sistema digestivo y su alimentación. http://www.faif.puc.cl. 2003.

Cummings, J.; Munnell, J. \& Vallenas, A. The mucigenous glandular mucosa in the complex stomach of two New-World Camelids, the llama and guanaco. J. Morph., 137:71-110, 1972.

Cunnimgham, G. Fisiología veterinaria. $2^{\mathrm{a}}$ ed. Secreción del tubo digestivo. McGraw-Hill, México, 1990. pp. 326-7.

Deeming, D, C. Introducción. En: El Avestruz: Biología, producción y sanidad. Acribia, Zaragoza, 2001. pp. 1-11.

Illanes, J.; Vigneaux, I. M.; Adaro, L.; Olivares, R.; Mendoza, J. \&
Riveros, V. Análisis morfológico comparado de la región fúndica entre un cánido (Canis familiaris) y un camélido suramericano (Lama glama). Rev. Chil. Anat., 12(2):161-7, 1994.

Illanes, J.; Fertilio, B.; Chamblas, M.; leyton, V. \& Verdugo, F. Descripción histológica de los diferentes segmentos del aparato digestivo de avestruz (Struthio camelus var. domesticus). Int. J. Morphol., 24(2):205-14, 2006.

McLelland, J. Atlas en color de Anatomía de las aves. En aparato digestivo. Interamericana, Buenos Aires, 1992. pp. 53-4.

Navarro, A. \& Benitez, H. Características generales de las aves. http://www.claseaves.8m.com, Julio, 2003.

Swart, D.; Rahn, H. \& de Kock, J. Nest microclimate and incubation water loss of eggs of the African ostrich (Struthio camelus var. domesticus). J. Exp. Zool. Supplement:239-46, 1987.

Direccion para Correspondencia:

Prof. Dr. Iulio Illanes Herrero.

Departamento de Morfologia,

Facultad de Medicina, Clinica Alemana-Universidad del Desarrollo

Av. Las Condes 12.438, Lo Barnechea.

Santiago

CHILE

Email: jillanes@udd.cl

Recibido : 03-02-2006

Aceptado: 24-06-2006 